\title{
BMJ Open Awareness and willingness towards pre- exposure prophylaxis against HIV infection among individuals seeking voluntary counselling and testing for HIV in Taiwan: a cross-sectional questionnaire survey
}

\author{
Yi-Chieh Lee, ${ }^{1}$ Sui-Yuan Chang, ${ }^{2,3}$ Kuan-Yin Lin, ${ }^{4}$ Lan-Hsin Chang, ${ }^{5}$ \\ Wen-Chun Liu, ${ }^{5}$ Cheng-Hsin Wu, ${ }^{5}$ Hsin-Yun Sun, ${ }^{5}$ Chien-Ching Hung, $, 5,6,7,8$ \\ Shan-Chwen Chang ${ }^{5}$
}

To cite: Lee Y-C, Chang S-Y, Lin K-Y, et al. Awareness and willingness towards pre-exposure prophylaxis against HIV infection among individuals seeking voluntary counselling and testing for HIV in Taiwan: a cross-sectional questionnaire survey. BMJ Open 2017;7:e015142. doi:10.1136/ bmjopen-2016-015142

- Prepublication history and additional material for this paper are available online. To view these files, please visit the journal online (http://dx.doi. org/10.1136/bmjopen-2016015142).

Received 12 November 2016 Revised 31 July 2017 Accepted 1 August 2017

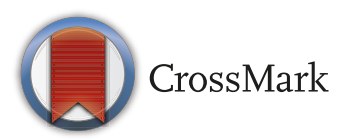

For numbered affiliations see end of article.

Correspondence to Dr Chien-Ching Hung; hcc0401@ntu.edu.tw

\section{ABSTRACT}

Objectives We aimed to investigate the awareness and willingness towards pre-exposure prophylaxis (PrEP) among individuals seeking voluntary counselling and testing (VCT) for HIV in Taiwan, where PrEP is currently not reimbursed by the insurance.

Methods Between April and October 2016, a questionnaire interview was conducted among VCT clients to inquire about the attitudes towards PrEP against HIV infection. Multivariate logistic regression analysis was performed to identify the associated factors with willingness to initiate PrEP.

Results During the 6-month period, 1173 VCT clients (99.8\%) completed the interviews, with $67.4 \%$ being homosexual or bisexual male. While $67.2 \%$ of the clients knew of postexposure prophylaxis, $40.2 \%$ heard of PrEP. Overall, 546 clients (46.5\%) were willing to initiate PrEP and $89.5 \%$ of them would choose event-driven PrEP. In multivariate analysis, male gender (OR $1.796 ; 95 \% \mathrm{Cl}$ 1.165 to 2.768 ), full-time job (OR $1.354 ; 95 \% \mathrm{Cl} 1.052$ to 1.742 ), one-night stand (OR $1.374 ; 95 \% \mathrm{Cl} 1.043$ to $1.810)$, having casual sex partners within 3 months $(O R$ $1.329 ; 95 \% \mathrm{Cl} 1.031$ to 1.714 ), condomless anal sex (OR $1.405 ; 95 \% \mathrm{Cl} 1.122$ to 1.878 ) and ever having chemsex or attending a drug party in the past 1 year (OR 2.571; $95 \% \mathrm{Cl} 1.541$ to 4.287$)$, regular screening for HIV infection (OR $1.321 ; 95 \% \mathrm{Cl} 1.021$ to 1.711 ) and knowledge of PrEP (OR $1.504 ; 95 \% \mathrm{Cl}, 1.159$ to 1.953$)$ were associated with willingness to initiate PrEP.

Conclusions Understanding the willingness to initiate PrEP against HIV among the VCT clients in Taiwan, which was associated with male gender, risky sexual behaviours and awareness of PrEP, will help inform the implementation of PrEP programme.

\section{INTRODUCTION}

Pre-exposure prophylaxis (PrEP), with the use of antiretroviral agents by HIV-negative individuals before potential exposure to HIV

\section{Strengths and limitations of this study}

This is the first study in the Asia-Pacific region to investigate the willingness of initiating pre-exposure prophylaxis (PrEP) among individuals, particularly men who have sex with men (MSM), who sought voluntary counselling and testing (VCT) for HIV.

- This study provides important information on factors and barriers associated with the willingness to start PrEP, which may inform the implementation of PrEP among individuals at risk for HIV infection in the Asia-Pacific region, where PrEP is not reimbursed by the national health insurance in most of the countries.

- Information and recall bias may limit the interpretation of the data that were obtained using questionnaire interviews from the clients.

- The findings in this single-centre study with an HIV incidence rate of 5.5 per 100 person-years among the VCT clients who are MSM may not be generalised to other settings, risk groups, centres or countries.

to prevent transmission, has been proven to be efficacious in the reduction of HIV transmission in clinical trials in certain populations, such as men who have sex with men (MSM), heterosexual men and women and injection drug users. ${ }^{1-4}$ The efficacy of PrEP in preventing HIV transmission depends on the adherence to daily doses of tenofovir disoproxil fumarate (TDF) and emtricitabine (FTC) ${ }^{5}$ which could be greater than $90 \%$ if the adherence is high (taking four tablets or more per week). ${ }^{5}$ The US Food and Drug Administration (FDA) has approved daily use of coformulated TDF/FTC as PrEP in July 2012. ${ }^{6}$ The US Centers for Disease Control and Prevention published a comprehensive 
clinical practice guideline of PrEP for the prevention of HIV infection in May 2014. ${ }^{7}$ Furthermore, the Pre-exposure Option for reducing HIV in the UK: immediate or Deferred (PROUD) study supported the addition of PrEP to the standard prevention strategies for MSM who were at risk of HIV infection in England, refuting the concerns of risk compensation, ${ }^{8}$ and the results of the Intervention Préventive de l'Exposition aux Risques avec et pour les Gays (IPERGAY) trial has provided another efficacious prevention strategy using on-demand PrEP in MSM against HIV infection in France. ${ }^{9}$

Although the results of the clinical trials of PrEP are promising and a significantly increasing trend in the utilisation of TDF/FTC for PrEP among commercially insured persons in the USA between 2010 and 2014, ${ }^{10}$ successful implementation of PrEP among high-risk populations needs an understanding of sociocultural relationship, knowledge and acceptability of the intervention and appropriate risk assessment. Several studies have explored the awareness and willingness of PrEP in different high-risk populations. ${ }^{11-13}$ The percentage of willingness and acceptability of PrEP ranged from $49.9 \%$ to $60.8 \%$ in these studies, which was associated with lower levels of education, regular gay scene attendance, highrisk unprotected anal intercourse (defined as having two or more partners, casual partners and/or unknown/ discordant partners in the previous 12 months) and testing for HIV or sexually transmitted infections (STIs) in the previous 12 months. ${ }^{12}$

In Asia-Pacific region, PrEP using TDF/FTC has not been widely implemented or reimbursed by the health insurance, except in Australia and Thailand. ${ }^{14}$ In Taiwan, 34479 cases of HIV infection have been reported to Taiwan Centers for Disease Control (CDC) between 1984 and 2016, with an estimated HIV prevalence of 1465 per 100000 populations. The annual cases of HIV infection continues to increase over the past decade and MSM remain the leading risk group of HIV infection, accounting for $60.4 \%$ of reported cases of HIV infection. ${ }^{15}$ HIV care, including combination antiretroviral therapy and monitoring of plasma HIV RNA load and CD4 count, is provided free-of-charge at designated hospitals around Taiwan. However, the National Health Insurance does not reimburse the cost related to PrEP, though Taiwan CDC and Taiwan AIDS Society have issued PrEP guidelines in May 2016. ${ }^{16}$

Understanding how PrEP is perceived by individuals at risk for HIV transmission is important before implementation of PrEP as one of the HIV prevention strategies. This cross-sectional questionnaire survey aimed to investigate the awareness and willingness toward PrEP among individuals seeking confidential voluntary counselling and testing (VCT) services for HIV in Taiwan.

\section{METHODS}

\section{Setting of the VCT services}

VCT services for HIV and syphilis have been offered free-of-charge in Taiwan, with grant support from the
Taiwan CDC. ${ }^{17}$ The National Taiwan University Hospital (NTUH) has provided VCT services since 1999, which was expanded in 2006. The number of attendees of the VCT programme at NTUH accounted for approximately $14 \%$ of the total number in Taiwan in recent years. ${ }^{18}{ }^{19}$ Each client seeking VCT services has a unique identification code for test results and has to complete a standardised, confidential, self-administered questionnaire interview prepared in Chinese (the English translated version available as online supplementary data at BMJ Open Online), designed by Taiwan CDC. After completion of counselling by trained counsellors, a blood sample was obtained for testing for HIV, syphilis and other STIs. This study was approved by the Research Ethics Committee of the hospital (registration number, 200904084R), and the VCT clients gave written informed consent with the use of the unique code before participating in this survey.

Among the VCT clients, the overall prevalence and incidence rate was $3.5 \%$ and 3.4 per 100 person-years (PY) for HIV infection, $2.2 \%$ and 1.6 per 100 PY for syphilis and $0.3 \%$ and 0.34 per $100 \mathrm{PY}$ for HCV infection, respectively, and the prevalence was $4.2 \%$ to $4.7 \%$ for chlamydia, $1.0 \%$ for amoebiasis and $0.7 \%$ for gonorrhoea. ${ }^{17} 1920$ Among the MSM population, the incidence rate of HIV infection (5.5 per $100 \mathrm{PY}$ ) was 10-fold higher than that of heterosexuals. ${ }^{21}$

\section{Self-administered questionnaire interview of PrEP}

The content of the questionnaire for VCT and PrEP comprised of four parts: first, the general and basic data of age, gender, level of education, current employment status and current monthly income status; second, frequency of seeking VCT services for HIV and activities clients engaged in, for example, one-night stand sex (defined as a single sexual encounter without an expectation of further relationship); third, risk assessment, which included the number of sexual partners in the past 3 months and condomless anal intercourse and use of recreational drugs in the past 1 year and fourth, the knowledge of HIV postexposure prophylaxis (PEP) and PrEP, willingness to use PrEP, concerns about using PrEP and reasons of no intention to use PrEP. PrEP was briefly described and information on the two current PrEP strategies was provided: oral PrEP with TDF/FTC administered on a once-daily basis and with TDF/FTC on an on-demand (event-driven) basis, especially on the differences of medication instruction and cost. ${ }^{8}$ During the study period, TDF/FTC had not yet obtained its approval for PrEP from Taiwan FDA. All antiretrovirals were only available at the designated hospitals for HIV care around Taiwan.

The pilot questionnaire interview was conducted among the first 66 VCT clients to identify the validity and comprehensibility of the questionnaire from 9 March to 7 April 2016. After modification of the questionnaire, 44 clients piloted questionnaire interview from 8 to 13 April 2016 and no changes were made to the questionnaire thereafter. 


\section{Statistical analysis}

Statistical analyses were performed using SAS software V.9.2 (SAS, North Carolina, USA). Continuous variables were reported as mean $\pm \mathrm{SD}$, analysed with non-parametric statistics and compared with Kruskal-Wallis tests. Categorical variables were expressed as percentage of the total number of clients analysed and compared with $\chi^{2}$ test. To identify factors associated with willingness of initiating PrEP, the variables with a $p$ value less than 0.05 in univariate analysis were entered into the multivariate logistic regression analysis. Model selection were conducted using a backward elimination technique based on two criteria (ie, Akaike Information Criteria (AIC) and Type III $p$ values) until the final model reached the optimum (minimum) AIC. ${ }^{22}$ A p value of less than 0.05 was considered statistically significant. Missing data were excluded for analysis.

\section{RESULTS}

From 8 April to 8 October 2016, a total of 1175 clients sought VCT services at NTUH and 1173 of them (99.8\%) agreed to participate in this study and completed questionnaire interviews. The demographic and clinical characteristics of the participants are shown in table 1 . The participants had a mean age of 29.7 years (SD, 7.9 years), $88.2 \%$ were of male gender, $67.4 \%$ were homosexual or bisexual male, $88.3 \%$ had diploma more than high school, $61.6 \%$ had full-time job and $57.2 \%$ had monthly income more than 30000 New Taiwan Dollars (NTDs) (approximately US\$950).

Regarding the activities that VCT clients were engaged in, $57.5 \%$ of the clients reported having sex with someone online from apps and $27.5 \%$ had one-night stand sex. Up to $80 \%$ of the clients $(79.7 \%$ ) reported having $1-5$ sexual partners in the past 3 months; $38.3 \%$ had condomless anal sex within the past 1 year and $11.1 \%$ had partners with HIV infection or other STIs. Sixty-nine percent of the clients had a casual sexual partner. Around $8.0 \%$ of the clients $(7.5 \%)$ admitted to use of recreational drug during sexual activity or attending a drug party within 3 months before this survey.

With respect to knowledge on HIV testing and prevention, $52.9 \%$ of the clients had used to attend regular screening for HIV. About two-thirds of the clients (67.2\%) knew of PEP while 40.2\% heard of PrEP before this survey. While $3.4 \%$ of the clients tested positive for HIV, $2.9 \%$ tested positive for syphilis in the survey during the study period.

Overall, 546 VCT clients (46.5\%) expressed their willingness to use PrEP, if TDF/FTC was approved for PrEP in Taiwan. Comparisons of the baseline data and characteristics between the clients with and without willingness to use PrEP are shown in table 1. Compared with individuals unwilling to use PrEP, individuals willing to use PrEP were more likely to be male $(93.4 \%$ vs $83.7 \%$, $\mathrm{p}<0.0001)$ and homosexual and bisexual male $(75.8 \%$ vs $60.0 \%, \mathrm{p}<0.0001)$, to have full-time job $(66.5 \%$ vs $58.2 \%$, $\mathrm{p}=0.0112)$ and current monthly income more than 30 000 NTDs $(60.9 \%$ vs $54.0 \%, \mathrm{p}=0.0203)$, to be engaged in sex with someone online from apps $(61.5 \%$ vs $53.9 \%$, $\mathrm{p}=0.0092)$ and with someone by dating at places like a pub, bathhouse or gym ( $11.5 \%$ vs $7.8 \%, \mathrm{p}=0.0362)$ and to have one-night stand sex ( $32.6 \%$ vs $23.1 \%, \mathrm{p}<0.0001)$.

When the risk behaviours were concerned, not having any sexual partners $(13.8 \%$ vs $19.7 \%, \mathrm{p}=0.0295)$ and having a casual partner $(53.7 \%$ vs $65.6 \%, \mathrm{p}<0.0001)$ were negatively associated with willingness to use PrEP. On the other hand, having condomless anal intercourse in the past 1 year $(46.2 \%$ vs $31.4 \%, \mathrm{p}<0.0001)$ and ever using recreational drug during sexual activity or attending a drug party in the past 1 year $(11.7 \%$ vs $3.8 \%, \mathrm{p}<0.0001)$ were associated with willingness to use PrEP.

Regarding the knowledge of HIV testing and prevention, regular screening for HIV (60.3\% vs $46.4 \%, \mathrm{p}<0.0001)$, and being aware of PEP $(73.1 \%$ vs $62.0 \%, \mathrm{p}<0.0001)$ and PrEP (48.0\% vs $33.3 \%, \mathrm{p}<0.0001)$ were more common in clients with willingness to use PrEP than those without. Compared with clients without willingness to use PrEP, clients with willingness to use PrEP were more likely to test positive for HIV during the current VCT visits $(5.7 \%$ vs $1.4 \%, \mathrm{p}<0.0001)$.

In multivariate analysis, factors associated with willingness to use PrEP are shown in table 2. Independent factors associated with willingness to start PrEP included male gender (OR 1.796; 95\% CI 1.165 to 2.768 ), having full-time job (OR 1.354; $95 \%$ CI 1.052 to 1.742), one-night stand sex (OR 1.374; 95\% CI 1.043 to 1.810 ), casual sex partners within 3 months (OR 1.329; 95\% CI 1.031 to 1.714 ) and condomless anal sex in the past 1 year (OR 1.405 ; $95 \%$ CI 1.122 to 1.878 ), ever using recreational drugs during sexual activity or attending a drug party in the past 1year (OR 2.571; 95\% CI 1.541 to 4.287 ) and having regular screening for HIV infection (OR 1.321; 95\% CI 1.021 to 1.711) and knowledge of PrEP (OR $1.504 ; 95 \%$ CI 1.159 to 1.953$)$.

Of the 546 clients who reported willingness to use PrEP, $89.5 \%$ preferred to use TDF/FTC on an on-demand (event-driven) basis. However, the concerns raised about PrEP included higher cost of medications (41.0\%), potential side effects $(33.5 \%)$ and inconvenient access to acquisition of TDF/FTC (28.7\%). The reasons for not considering PrEP for the 627 clients included having adopted protective measures during sex $(78.5 \%)$ and concerns about higher cost of medications (18.5\%), potential side effects $(11.6 \%)$ and inconvenient access to acquisition of TDF/FTC (8.6\%).

\section{DISCUSSION}

This is the first study to report on awareness of using TDF/ FTC as PrEP against HIV and willingness to use PrEP among individuals seeking for VCT in the Asia-Pacific region in recent years. We found that, while PrEP was not reimbursed by the National Health Insurance in Taiwan, $46.5 \%$ of VCT clients were willing to use PrEP and $89.5 \%$ 
Table 1 Univariate analysis of factors associated with the willingness to use PrEP among 1173 individuals seeking voluntary counselling testing for HIV in Taiwan

\begin{tabular}{|c|c|c|c|c|}
\hline Variables & All individuals & $\begin{array}{l}\text { Individuals willing to } \\
\text { use PrEP }\end{array}$ & $\begin{array}{l}\text { Individuals unwilling } \\
\text { to use PrEP }\end{array}$ & $\begin{array}{l}\text { Statistics } \\
\text { p value* }\end{array}$ \\
\hline Patient number, $\mathrm{N}(\%)$ & $1173(100.0)$ & $546(46.5)$ & $627(53.5)$ & \\
\hline Age, mean (SD), years & $29.7(7.9)$ & $29.7(7.2)$ & $29.8(8.6)$ & 0.2643 \\
\hline \multicolumn{5}{|l|}{ Gender, \% } \\
\hline Male & 88.2 & 93.4 & 83.7 & \multirow[t]{3}{*}{$<0.0001$} \\
\hline Female & 11.8 & 6.6 & 16.3 & \\
\hline Transgender & 0.0 & 0.0 & 0.0 & \\
\hline Highest level of education, $\%$ & 100.0 & 100.0 & 100.0 & \\
\hline More than high school & 88.3 & 89.1 & 87.7 & \multirow[t]{2}{*}{0.5241} \\
\hline High school or less & 11.7 & 11.0 & 12.3 & \\
\hline Current employment status, $\%(n / N)$ & 99.5 & 99.5 & 99.5 & \\
\hline Full time & $61.6(719 / 1167)$ & $66.5(356 / 543)$ & $58.2(363 / 624)$ & 0.0112 \\
\hline Others & $38.4(448 / 1167)$ & $34.4(187 / 543)$ & $41.8(261 / 624)$ & \\
\hline \multicolumn{5}{|l|}{ Current monthly income status, \% $(n / N)$} \\
\hline$<30000$ NTDs & $42.8(497 / 1161)$ & $39.1(210 / 537)$ & $46.0(287 / 624)$ & \multirow[t]{2}{*}{0.0203} \\
\hline$\geq 30000$ NTDs & $57.2(664 / 1161)$ & $60.9(327 / 537)$ & $54.0(337 / 624)$ & \\
\hline \multicolumn{5}{|l|}{ Sexual partners, \% } \\
\hline Non-MSM male & 20.9 & 17.6 & 23.8 & \multirow[t]{3}{*}{$<0.0001$} \\
\hline MSM or bisexual male & 67.4 & 75.8 & 60.0 & \\
\hline Female & 11.8 & 6.6 & 16.3 & \\
\hline \multicolumn{5}{|l|}{ Activities engaged in, $\%$} \\
\hline Sex work (provider or consumer) & 10.1 & 9.5 & 10.7 & 0.5611 \\
\hline $\begin{array}{l}\text { Having sex with someone dating } \\
\text { online or from apps }\end{array}$ & 57.5 & 61.5 & 53.9 & 0.0092 \\
\hline $\begin{array}{l}\text { Having sex with someone dating at } \\
\text { places, like a pub, bathhouse or gym }\end{array}$ & 9.5 & 11.5 & 7.8 & 0.0362 \\
\hline Attending a sex party & 0.5 & 0.7 & 0.3 & 0.4255 \\
\hline One-night stand sex & 27.5 & 32.6 & 23.1 & $<0.0001$ \\
\hline \multicolumn{5}{|l|}{ Risk behaviours, \% (n/N) } \\
\hline \multicolumn{5}{|l|}{ Number of sex partners within 3 months } \\
\hline 0 & $16.9(198 / 1169)$ & $13.8(75 / 543)$ & $19.7(123 / 626)$ & \multirow[t]{3}{*}{0.0295} \\
\hline $1-5$ & $79.7(932 / 1169)$ & $82.7(449 / 543)$ & $77.2(483 / 626)$ & \\
\hline$>5$ & $3.3(39 / 1169)$ & $3.5(19 / 543)$ & $3.2(20 / 626)$ & \\
\hline $\begin{array}{l}\text { Having a committed sexual partner } \\
\text { within } 3 \text { months }\end{array}$ & $45.2(530 / 1173)$ & $47.6(260 / 546)$ & $43.1(270 / 627)$ & 0.1263 \\
\hline $\begin{array}{l}\text { Having a casual sex partner within } \\
3 \text { months }\end{array}$ & $60.0(704 / 1173)$ & $53.7(293 / 546)$ & $65.6(411 / 627)$ & $<0.0001$ \\
\hline $\begin{array}{l}\text { Condomless anal sex in the past } \\
1 \text { year }\end{array}$ & $38.3(449 / 1173)$ & $46.2(252 / 546)$ & $31.4(197 / 627)$ & $<0.0001$ \\
\hline $\begin{array}{l}\text { Partner infected with HIV or other } \\
\text { STIs }\end{array}$ & $11.1(130 / 1173)$ & $12.6(69 / 546)$ & $9.7(61 / 627)$ & 0.1355 \\
\hline Ever having STIs in the past 1 year & $6.8(80 / 1173)$ & $8.2(45 / 546)$ & $5.6(35 / 627)$ & 0.0815 \\
\hline $\begin{array}{l}\text { Alcohol consumption before or during } \\
\text { sexual activity }\end{array}$ & $17.3(203 / 1173)$ & $17.0(93 / 546)$ & $17.5(110 / 627)$ & 0.8771 \\
\hline $\begin{array}{l}\text { Ever using recreational drugs before } \\
\text { or during sexual activity or attending } \\
\text { drug party in the past } 1 \text { year }\end{array}$ & $7.5(88 / 1173)$ & $11.7(64 / 546)$ & $3.8(24 / 627)$ & $<0.0001$ \\
\hline
\end{tabular}


Table 1 Continued

\begin{tabular}{|c|c|c|c|c|}
\hline Variables & All individuals & $\begin{array}{l}\text { Individuals willing to } \\
\text { use PrEP }\end{array}$ & $\begin{array}{l}\text { Individuals unwilling } \\
\text { to use PrEP }\end{array}$ & $\begin{array}{l}\text { Statistics } \\
\text { p value* }\end{array}$ \\
\hline \multicolumn{5}{|l|}{ Knowledge on prevention, \% } \\
\hline Knew of HIV PEP & 67.2 & 73.1 & 62.0 & $<0.0001$ \\
\hline Knew of HIV PrEP & 40.2 & 48.0 & 33.3 & $<0.0001$ \\
\hline HIV & $3.4(40 / 1173)$ & $5.7(31 / 546)$ & $1.4(9 / 627)$ & $<0.0001$ \\
\hline
\end{tabular}

*p Value was calculated for the differences among the two groups. Continuous variables were analysed with non-parametric statistics, Kruskal-Wallis test, while categorical variables with $\chi^{2}$ test.

MSM, men who have sex with men; NTD, New Taiwan Dollar; PEP, postexposure prophylaxis; PrEP, pre-exposure prophylaxis; STI, sexually transmitted infection; VCT, voluntary counselling testing.

of them preferred to use PrEP on an event-driven basis. VCT clients who were male with full-time job, regular screening for HIV infection and knowledge of PrEP and engaged in one-night stand sex, having casual sex partners and condomless anal sex and using recreational drugs before or during sex were more likely to report willingness of initiation of PrEP. Given the demonstrated efficacy of PrEP in preventing HIV infection among MSM who were engaged in risky behaviours, ${ }^{89}$ these findings suggest that there is a substantial unmet need for a new prevention strategy against HIV transmission among individuals who perceived themselves at risk for HIV and STIs and sought VCT in Taiwan.

In this study, the risky behaviours that we identified to be associated with willingness to use PrEP, particularly condomless anal sex, were similar to those reported in other studies, ${ }^{12} 23-27$ which implies that engagement in risky sexual behaviours may potentiate the willingness to use PrEP. However, ever using recreational drugs before or during sexual activity has only been identified in a recent study that revealed the association between use of amyl nitrate and having sex outside the relationship. ${ }^{28}$ Recreational drug use before or during sex, also termed 'chemsex', was associated with increased odds of unprotected anal intercourse by encounter-level analysis ${ }^{29}{ }^{30}$ which has been found to be a risk factor for acquisition of HIV infection. ${ }^{31} 32$ Therefore, the published guidelines also identify those persons having chemsex as potential candidates to initiate PrEP. ${ }^{7}$

Regarding the awareness of HIV prevention, we found that prior knowledge of PrEP was associated with willingness to uptake PrEP. Goedel and colleagues also identified the correlation between awareness with willingness to use PrEP in gay, bisexuals and other MSM in New York City. ${ }^{33}$ In Spain, MSM who had heard of PrEP were more forceful in their options on the willingness to use PrEP. ${ }^{34}$ Therefore, with the increased awareness of PrEP, the population at risk will be more likely to accept and initiate PrEP. To effectively implement PrEP programme, information, education and counselling of PrEP should be delivered to the populations at risk for HIV infection.

Among VCT clients with willingness to use PrEP, almost $90 \%$ of them preferred event-driven strategy to prevention against HIV infection in our study. However, the VCT clients were concerned about inconvenient access to acquisition of TDF/FTC (28.7\%), higher cost of the medications $(41.0 \%)$ and their potential side effects $(33.5 \%)$. In the Dutch study, Bil and colleagues found that

Table 2 Multivariate analysis for factors associated with the willingness to use pre-exposure prophylaxis (PrEP) against HIV infection in individuals seeking voluntary counselling and testing for HIV

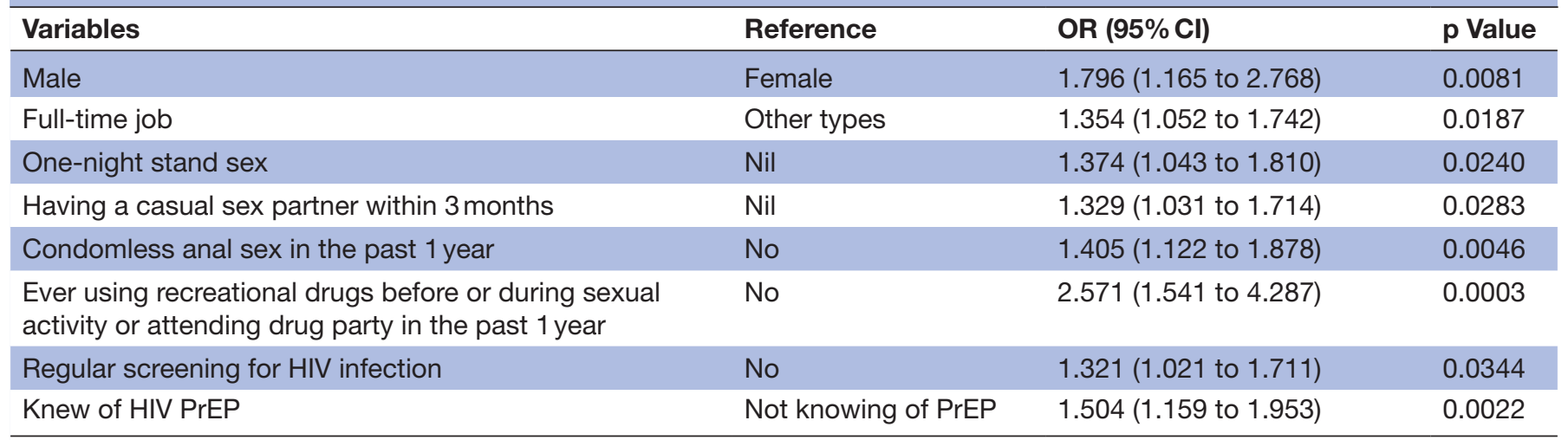

NTD, New Taiwan Dollar; PEP, postexposure prophylaxis. 
compared with daily PrEP use, the benefits of intermittent PrEP use included the lower cost and potential risk of side effects and lower threshold to start PrEP, while the barriers to PrEP included the perceived need to plan their sex life and adherence to multiple prevention strategies among MSM. ${ }^{35}$ Another qualitative research identified preference of on-demand PrEP over daily PrEP, injectable PrEP and free or standardised access through community organisations or government hospitals among MSM in India. ${ }^{36}$ The greatest consensus regarding more acceptable PrEP attributes has been in the mode of delivery and its cost. ${ }^{37}$ Therefore, provision of information on PrEP efficacy and potential side effects, a convenient access to PrEP service and reimbursement of PrEP are necessary to make implementation of PrEP more successful among the populations at risk.

The cost of PrEP medications has been a major concern and barrier to wide implementation of PrEP among persons at risk for HIV infection. Several mathematical models investigated the cost-effectiveness or impact of PrEP on the HIV epidemic among MSM in North America, ${ }^{38-41}$ Australia, ${ }^{42}$ the $\mathrm{UK}^{43}$ and the Netherlands. ${ }^{44}$ All these studies demonstrated that targeting PrEP to MSM at high risk of HIV infection was cost-effective. The variability of cost-effectiveness among the different studies could be due to the differences in the HIV epidemic among MSM, direct and indirect costs and the percentage of MSM targeted for intervention. Therefore, the demonstration of the cost-effectiveness of PrEP and the potential impact on HIV epidemic when PrEP is used along with other HIV treatment and prevention programmes should be able to provide evidence and rationale for the policymakers to commit to the implementation of affordable PrEP for persons at risk for HIV infection. ${ }^{3738}$

Compared with other studies about willingness to use PrEP, our study had a large sample size (1173 participants) and high participation rate of questionnaire interview (99.8\% of 1175 VCT clients approached). However, several limitations should be considered. First, the study was conducted using confidential questionnaire interview to collect information on sexual risk behaviours and all data were self-reported; therefore, it is difficult to avoid recall bias and we were not able to verify the information provided. Second, the willingness of PrEP use might be overestimated because of the hypothetical questions in questionnaire interview. Given the barriers to initiation of PrEP using TDF/FTC, it remains to be seen whether action will be taken by the individuals with risky behaviours when TDF/FTC was approved for PrEP in Taiwan. Third, this is a single-centre study including VCT clients who were mainly MSM with an HIV incidence rate of 5.5 per 100 PY and all the study participants are of Asia ethnicity. Therefore, our findings may not be generalised to participants of other ethnicities and countries.

\section{CONCLUSIONS}

Among the individuals seeking VCT services in Taiwan, a substantial portion $(46.5 \%)$ of the participants had willingness to initiate PrEP against HIV infection, which were independently associated with having risky sexual behaviours and awareness of PrEP. The barriers to PrEP included cost, potential adverse effects and accessibility issues. The implementation of PrEP in Taiwan could be facilitated through dissemination of the information on PrEP and provision of PrEP that is affordable and easy to access.

\section{Author affiliations}

${ }^{1}$ Department of Internal Medicine, Lotung Poh-Ai Hospital, Medical Lo-Hsu Foundation, I-Lan, Taiwan

${ }^{2}$ Department of Clinical Laboratory Sciences and Medical Biotechnology, National Taiwan University College of Medicine, Taipei, Taiwan

${ }^{3}$ Department of Laboratory Medicine, National Taiwan University Hospital and National Taiwan University College of Medicine, Taipei, Taiwan

${ }^{4}$ Department of Medicine, National Taiwan University Hospital Jin-Shan Branch, New Taipei City, Taiwan

${ }^{5}$ Department of Internal Medicine, National Taiwan University Hospital and National Taiwan University College of Medicine, Taipei, Taiwan

${ }^{6}$ Department of Parasitology, National Taiwan University College of Medicine, Taipei, Taiwan

${ }^{7}$ Department of Medical Research, China Medical University Hospital, Taichung, Taiwan

${ }^{8}$ China Medical University, Taichung, Taiwan

Acknowledgements We would like to thank the VCT clients for their participation in this survey and Taiwan Centers for Disease Control for the research grant support.

Contributors YCL, SYC, HYS, and CCH designed the study; KYL, LSC, WCL and CHW performed the questionnaire interview and data collection; YCL, HYS, KYL and WCL contributed to data analysis and SCC oversaw the study. First draft was written by YCL with substantive revisions and input from all authors. All authors have read and approved the final manuscript.

Funding This study was supported by grants from Taiwan Centers for Disease Control (MOHW105-CDC-C-114-000104 to CCH). The funding source played no role in study design and conduct, data collection, analysis or interpretation, writing of the manuscript or the decision to submit it for publication.

Competing interests $\mathrm{CCH}$ has received research support from Janssen, Merck, Bristol-Myers Squibb and ViiV; speaker honoraria from ViiV, Abbvie and Gilead and served on advisory boards for Gilead, Janssen, ViiV and Abbvie. Other authors, none to declare.

Ethics approval Research Ethics Committee of National Taiwan University Hospital Provenance and peer review Not commissioned; externally peer reviewed.

Data sharing statement № additional data are available.

Open Access This is an Open Access article distributed in accordance with the Creative Commons Attribution Non Commercial (CC BY-NC 4.0) license, which permits others to distribute, remix, adapt, build upon this work non-commercially, and license their derivative works on different terms, provided the original work is properly cited and the use is non-commercial. See: http://creativecommons.org/ licenses/by-nc/4.0/

(c) Article author(s) (or their employer(s) unless otherwise stated in the text of the article) 2017. All rights reserved. No commercial use is permitted unless otherwise expressly granted.

\section{REFERENCES}

1. Grant RM, Lama JR, Anderson PL, et al. Preexposure chemoprophylaxis for HIV prevention in men who have sex with men. N Engl J Med 2010;363:2587-99.

2. Baeten JM, Donnell D, Ndase P, et al. Antiretroviral prophylaxis for HIV prevention in heterosexual men and women. $N$ Engl J Med 2012;367:399-410. 
3. Thigpen MC, Kebaabetswe PM, Paxton LA, et al. Antiretroviral preexposure prophylaxis for heterosexual HIV transmission in Botswana. N Engl J Med 2012;367:423-34.

4. Choopanya K, Martin M, Suntharasamai P, et al. Antiretroviral prophylaxis for HIV infection in injecting drug users in Bangkok, Thailand (the Bangkok Tenofovir Study): a randomised, double-blind, placebo-controlled phase 3 trial. Lancet 2013;381:2083-90.

5. Grant RM, Anderson PL, McMahan V, et al. Uptake of pre-exposure prophylaxis, sexual practices, and HIV incidence in men and transgender women who have sex with men: a cohort study. Lancet Infect Dis 2014;14:820-9.

6. United States Food and Drug Administration. FDA approves first drug for reducing the risk of sexually acquired HIV infection. 2012 http://www.fda.gov/NewsEvents/Newsroom/PressAnnouncements/ ucm312210.htm (accessed 10 Aug 2016).

7. United States Public Health Service. Preexposure prophylaxis for the prevention of HIV in the United States-2014: a clinical practice guideline. http://www.cdc.gov/hiv/pdf/guidelines/ PrEPguidelines2014.pdf (accessed 10 Aug 2016).

8. McCormack S, Dunn DT, Desai M, et al. Pre-exposure prophylaxis to prevent the acquisition of HIV-1 infection (PROUD): effectiveness results from the pilot phase of a pragmatic open-label randomised trial. Lancet 2016;387:53-60.

9. Molina JM, Capitant C, Spire B, et al. On-demand preexposure prophylaxis in men at high risk for HIV-1 infection. $N$ Engl $J$ Med 2015;373:2237-46.

10. Wu H, Mendoza MC, Huang YA, et al. Uptake of HIV preexposure prophylaxis among commercially insured persons - United States, 2010-2014. Clin Infect Dis 2017:64:144-9.

11. Hoots BE, Finlayson T, Nerlander L, et al. Willingness to take, use of, and indications for pre-exposure prophylaxis among men who have sex with men - 20 US Cities, 2014. Clin Infect Dis 2016;63:672-7

12. Young I, Li J, McDaid L. Awareness and willingness to use HIV pre-exposure prophylaxis amongst gay and bisexual men in Scotland: implications for biomedical HIV prevention. PLoS One 2013;8:e64038.

13. Hoff CC, Chakravarty D, Bircher AE, et al. Attitudes towards PrEP and anticipated condom use among concordant HIV-negative and HIV-discordant male couples. AIDS Patient Care STDS 2015;29:408-17.

14. Zablotska I, Grulich AE, Phanuphak N, et al. PrEP implementation in the Asia-Pacific region: opportunities, implementation and barriers. $J$ Int AIDS Soc 2016;19:21119.

15. Taiwan CDC. Statistics of HIV/AIDS. 2016 http://www.cdc.gov.tw/ downloadfile. aspx? fid=CF697A305BCC94CF (accessed 1 May 2017).

16. Taiwan AIDS society. Guideline for the use of pre-exposure oral prophylaxis (PrEP) in Taiwan. $2016 \mathrm{http}: / /$ www.aids-care.org.tw/\% E8\%87\%BA\%E7\%81\%A3\%E6\%9A\%B4\%E9\%9C\%B2\%E5\% 89\%8D\%E5\%8F\%A3\%E6\%9C\%8D\%E9\%A0\%90\%E9\%98\%B2\% E6\%80\%A7\%E6\%8A\%95\%E8\%97\%A5\%E4\%BD\%BF\%E7\% 94\%A8\%E6\%8C\%87\%E5\%BC\%95(\%E5\%85\%AC\%E5\%91\%8A $\%$ E7\%89\%8820160502).pdf (accessed 17 May 2017).

17. Wu H, Wu PY, Li SY, Py W, Sy L, et al. Maximising the potential of voluntary counselling and testing for HIV: sexually transmitted infections and HIV epidemiology in a population testing for HIV and its implications for practice. Sex Transm Infect 2012;88:612-6.

18. Lai CC, Liu WC, Fang CT, et al. Transmitted drug resistance of HIV-1 strains among individuals attending voluntary counselling and testing in Taiwan. J Antimicrob Chemother 2016;71:226-34.

19. Tsai JC, Hung CC, Chang SY, et al. Increasing incidence of recent hepatitis $C$ virus infection among persons seeking voluntary counselling and testing for HIV and sexual transmitted infections in Taiwan. BMJ Open 2015;13:e008406.

20. Yang CJ, Li SY, Chang SY, et al. Associated factors with and genotypes of Chlamydia trachomatis infection among clients seeking voluntary counseling and testing for HIV infection in Taiwan. $J$ Microbiol Immunol Infect 2014;47:526-32.

21. Liu WC, Chang LH, Wu PY, Py W, et al. Seroincidence of HIV and prevalence of transmitted drug resistance of HIV-1 strains among persons seeking voluntary counselling and testing in Taiwan. $J$ Int AIDS Soc 2014;17:19758

22. Lima VD, Geller J, Bangsberg DR, et al. The effect of adherence on the association between depressive symptoms and mortality among HIV-infected individuals first initiating HAART. AIDS 2007:21:1175-83.

23. Saberi P, Gamarel KE, Neilands TB, et al. Ambiguity, ambivalence, and apprehensions of taking HIV-1 pre-exposure prophylaxis among male couples in San Francisco: a mixed methods study. PLoS One 2012; 7:e50061.

24. Krakower DS, Mimiaga MJ, Rosenberger JG, et al. Limited awareness and low immediate uptake of pre-exposure prophylaxis among men who have sex with men using an internet social networking site. PLoS One 2012;7:e33119.

25. Grov $\mathrm{C}$, Whitfield TH, Rendina HJ, et al. Willingness to take PrEP and potential for risk compensation among highly sexually active gay and bisexual men. AIDS Behav 2015;19:2234-44.

26. Hoagland B, De Boni RB, Moreira Rl, et al. Awareness and willingness to use pre-exposure prophylaxis (PrEP) among men who have sex with men and transgender women in Brazil. AIDS Behav 2017;:21:1278-87.

27. Holt M, Lea T, Schmidt HM, et al. Willingness to use and have sex with men taking HIV pre-exposure prophylaxis (PrEP): results of online surveys of Australian gay and bisexual men, 2011-2015. Sex Transm Infect 2017;93::438-44.

28. Mitchell JW, Stephenson R. HIV-negative partnered men's willingness to use pre-exposure prophylaxis and associated factors among an internet sample of U.S. HIV-negative and HIV-discordant male couples. LGBT Health 2015;2:35-40.

29. Boone MR, Cook SH, Wilson P. Substance use and sexual risk behavior in HIV-positive men who have sex with men: an episodelevel analysis. AIDS Behav 2013;17:1883-7.

30. Melendez-Torres GJ, Hickson F, Reid D, et al. Nested event-level case-control study of drug use and sexual outcomes in multipartner encounters reported by Men who have sex with men. AIDS Behav 2016;20:646-54.

31. Plankey MW, Ostrow DG, Stall R, et al. The relationship between methamphetamine and popper use and risk of HIV seroconversion in the multicenter AIDS cohort study. J Acquir Immune Defic Syndr 2007;45:85-92.

32. Chen X, Li X, Zheng J, et al. Club drugs and HIV/STD infection: an exploratory analysis among men who have sex with men in Changsha, China. PLoS One 2015;10:e0126320.

33. Goedel WC, Halkitis PN, Greene RE, et al. Correlates of awareness and willingness to use pre-exposure prophylaxis (PrEP) in gay, bisexual, and other men who have sex with men who use geosocialnetworking smartphone applications in New York City. AIDS Behav 2016;20:1435-42.

34. Ferrer L, Folch C, Fernandez-Davila P, et al. Awareness of preexposure prophylaxis for HIV, willingness to use it and potential barriers or facilitators to uptake among men who have sex with men in Spain. AIDS Behav 2016;20:1423-33.

35. Bil JP, van der Veldt WM, Prins M, et al. Motives of Dutch men who have sex with men for daily and intermittent HIV pre-exposure prophylaxis usage and preferences for implementation: a qualitative study. Medicine 2016;95:e4910.

36. Chakrapani V, Newman PA, Shunmugam M, et al. Acceptability of HIV pre-exposure prophylaxis (PrEP) and implementation challenges among men who have sex with men in India: a qualitative investigation. AIDS Patient Care STDS 2015;29:569-77.

37. Kessler J, Myers JE, Nucifora KA, et al. Evaluating the impact of prioritization of antiretroviral pre-exposure prophylaxis in New York. AIDS 2014;28:2683-91.

38. Koppenhaver RT, Sorensen SW, Farnham PG, et al. The costeffectiveness of pre-exposure prophylaxis in men who have sex with men in the United States: an epidemic model. J Acquir Immune Defic Syndr 2011;58:e51-e52.

39. Ross EL, Cinti SK, Hutton DW, et al. clinically actionale strategy for targeting HIV preexposure prophylaxis to high risk men who have sex with men. J Acquir Immune Defic Syndr 2016;72:e61-7.

40. Juusola JL, Brandeau ML, Owens DK, et al. The cost-effectiveness of preexposure prophylaxis for HIV prevention in the United States in men who have sex with men. Ann Intern Med 2012;156:541-50.

41. MacFadden DR, Tan DH, Mishra S. Optimizing HIV pre-exposure prophylaxis implementation among men who have sex with men in a large urban centre: a dynamic modelling study. J Int AIDS Soc 2016;23:20791.

42. Schneider K, Gray RT, Wilson DP. A cost-effectiveness analysis of HIV preexposure prophylaxis for men who have sex with men in Australia. Clin Infect Dis 2014;58:1027-34.

43. Cambiano V, Miners A, Dunn DT, et al. Is PrEP for HIV prevention cost-effective in MSM in the UK? BASHH Spring Conference, Glasgow, UK. 2015. Sex Transm Infect 2015;91:A1.

44. Nichols BE, Boucher CAB, van der Valk M, et al. Cost-effectiveness analysis of pre-exposure prophylaxis for HIV-1 prevention in the Netherlands: a mathematical modelling study. Lancet Infect Dis 2016;16:1423-9. 